\title{
Implications of Vector Boson Scattering Unitarity in Composite Higgs Models
}

\author{
Diogo Buarque Franzosi \\ Georg-August-Universität Göttingen \\ E-mail: dbuarquegwdg. de
}

The strong nature of Composite Higgs models manifests at high energies through the growing behavior of the scattering amplitudes of longitudinally polarized weak bosons that leads to the formation of composite resonances as well as non-resonant strong effects. In this talk the unitarity of these scattering amplitudes is used as a tool to assess the profile of the lightest CP-even scalar composite excitation and analyze its eventual signature at hadron colliders. This paper is based on the more complete study of Ref [1], in which non-resonant and vector composite resonances are also studied.

EPS-HEP 2017, European Physical Society conference on High Energy Physics 5-12 July 2017

Venice, Italy 


\section{Introduction}

The strong nature of the Nambu-Goldstone bosons (NGB) in Composite Higgs (CH) models manifests in the Goldstone Boson Scattering GBS through the miscancellation of Feynman diagrams and the divergent behavior of the scattering amplitudes according to the Low Energy Theorems (LET)

$$
\mathscr{A}(\pi \pi \rightarrow \pi \pi) \sim \frac{s}{f^{2}}=\frac{s}{v^{2}} \sin ^{2} \theta,
$$

where $f$ is the NGB decay constant associated to the condensate and $\theta$ is the misalignment angle between the condensate expectation value and the vacuum that break EW symmetry. The growing behavior of GBS amplitudes must be eventually controlled by strong effects at high energies, either in the form of broad continuum enhancements or in the form of composite resonances, saturating unitarity similarly to what happens in hadron physics.

Through the analysis of the GBS amplitudes and under the guidance of unitarity principles we will set limits on the scale of resonance formation in the scalar channel which is only poorly described by lattice calculations. We will show that near the scale of leading-order (LO) unitarity violation the continuum of LET dominates the scattering amplitudes and prevents the formation of Breit-Wigner resonances. We will also argue that we can not only set constraints on the masses of resonances, but also on their couplings, if we assume basic criteria of saturation of unitarity and analyticity provided by the Inverse Amplitude Method (IAM) of unitarization.

Following our assessment of resonance profiles via the study of unitarization of GBS amplitudes, we will estimate in a realistic collider environment the potential to observe scalar-resonant scenarios of strong VBS in $p p \rightarrow j j Z Z \rightarrow j j 4 \ell$ channel.

\section{Fundamental Minimal CH Model (FMCHM)}

We use the FMCHM as template of $\mathrm{CH}$ model based on the coset $\mathrm{SU}(4) / \mathrm{Sp}(4)$, which is the simplest global symmetry breaking pattern which can be realized in terms of an underlying fermionic gauge theory $[2,3]$.

The value of the misalignment angle $\theta$ is typically a good parametrization of fine-tuning [4,3], thus we expect it to be not too small. On the other hand, large angles are not favored by data due to deviations of the Higgs couplings from the SM predictions, which upset EW precision observables (EWPO) resulting in an upper bound $\sin \theta \lesssim 0.2[5]$

We use the Callan-Coleman-Wess-Zumino (CCWZ) construction $[6,7]$ to write the effective Lagrangian. The lowest dimension $(d=2)$ term is given by

$$
\mathscr{L}_{2}=\frac{1}{2} f^{2}\left\langle x_{\mu} x^{\mu}\right\rangle
$$

where $x_{\mu}$ is the projection of the Maurer-Cartan form containing the 5 NGBs and \langle\rangle is the trace.

In order to analyze unitarity it is imperative to include higher order terms due to the strong relation between perturbativity and unitarity. Since the CCWZ Lagrangian is an effective nonrenormalizable theory, each order in the perturbation expansion has to be accompanied by a tower 
of higher dimension operators in order to carry out the renormalization program. The $d=4 \mathrm{La}-$ grangian is given by

$$
\mathscr{L}_{4}=L_{0}\left\langle x^{\mu} x^{v} x_{\mu} x_{v}\right\rangle+L_{1}\left\langle x^{\mu} x_{\mu}\right\rangle\left\langle x^{v} x_{v}\right\rangle+L_{2}\left\langle x^{\mu} x^{v}\right\rangle\left\langle x_{\mu} x_{v}\right\rangle+L_{3}\left\langle x^{\mu} x_{\mu} x^{v} x_{v}\right\rangle .
$$

In addition, we assume that the CP-even scalar excitation is the lightest in the composite spectrum. Additional scalars are a common feature in composite extensions of the SM, see e.g. [8, 4]. The scalar singlet $\sigma$ can be incorporated in a simple general way

$$
\mathscr{L}_{\sigma}=\frac{1}{2} \kappa(\sigma / f) f^{2}\left\langle x_{\mu} x^{\mu}\right\rangle+\frac{1}{2} \partial_{\mu} \sigma \partial^{\mu} \sigma-\frac{1}{2} M_{\sigma}^{2} \sigma^{2},
$$

with $\kappa(\sigma / f)=1+\kappa^{\prime} \sigma / f+\kappa^{\prime \prime} \sigma^{2} /\left(2 f^{2}\right)+\cdots$. The potential (which must be added to $\mathscr{L}_{\sigma}$ ) generates mixing between $\sigma$ and the Higgs $h$ which can be neglected at the high energies we are interested in.

\section{Unitarity Implications}

After expanding the $\pi \pi \rightarrow \pi \pi$ elastic scalar scattering amplitude, $\mathscr{A}(s, t)$, in partial waves $a_{J}(s)$, the unitarity condition reads $\operatorname{Im} a_{J}(s)=\left|a_{J}(s)\right|^{2}$. It is however useful to define a less strong criteria of unitarity, which holds also in a perturbative expansion and when inelastic channels are open,

$$
|a(s)|<1 \text {. }
$$

Similarly to the expansion in isospin of pion scattering, the $2 \rightarrow 2$ NGB scattering in $\mathrm{SU}(4) / \mathrm{Sp}(4)$ can be expanded in definite multiplets of $\mathrm{Sp}(4)$, as $\mathbf{5} \otimes \mathbf{5}=\mathbf{1} \oplus \mathbf{1 0} \oplus \mathbf{1 4}$. From the $d=2$ Lagrangian (eq. (2.1)) we can get the LO amplitudes. In this report, we will consider only the singlet scalar channel $\mathbf{1} \equiv A$ with partial wave amplitude given by

$$
a_{A 0}^{(0)}(s)=\frac{s}{16 \pi f^{2}} .
$$

Therefore, according to eq. (3.1) unitarity is fated to be violated at energies

$$
\sqrt{s} \gtrsim 4 \sqrt{\pi} f
$$

Since from EWPO we expect $\sin \theta \lesssim 0.2$, we need to reach partonic energies at least of the order of $\sqrt{s} \sim 8 \mathrm{TeV}$ to observe strong VBS effects. Such energies could in principle be at the extreme corner of LHC potential, but it seems more feasible to be reached at a higher energy machine, such as a $100 \mathrm{TeV}$ collider. Even for lower angles, e.g. $\theta=0.1$, unitarity violation would take place around $\sqrt{s} \sim 16 \mathrm{TeV}$, which is within the reach of a $100 \mathrm{TeV}$ machine. If inelastic channels are open, the scale of strong effects are expected to be lower.

The next-to-leading order (NLO) correction to the partial wave amplitudes, which includes the tree level diagrams involving dimension-6 operators, eq. (2.2), and one-loop diagrams, is given by

$$
a_{A 0}^{(1)}(s)=\frac{s^{2}}{32 \pi f^{4}}\left[\frac{1}{16 \pi^{2}}\left(\frac{29}{12}+\frac{46}{18} \log \left(\frac{s}{\mu^{2}}\right)+2 \pi i\right)+\frac{2}{3} \widehat{L_{A}}(\mu)\right]
$$


with $\widehat{L_{A}}(\mu)=\widehat{L_{0}}(\mu)+68 \widehat{L_{1}}(\mu)+36 \widehat{L_{2}}(\mu)+17 \widehat{L_{3}}(\mu)$ and $\widehat{L}(\mu)$ is the renormalized effective coefficient in the $\overline{M S}$ scheme at the scale $\mu$.

The NLO and LO partial wave amplitudes can be defined as $a^{N L O}(s)=a^{(0)}(s)+a^{(1)}(s)$ and $a^{L O}(s)=a^{(0)}(s)$. The growing behavior of these amplitudes eventually leads to the violation of unitarity, meaning that the perturbative expansion is jeopardized and strong effects must appear to tame the amplitudes. It is important to note that we have found the NLO corrections always anticipate the violation of unitarity to lower scales, thus the scale $\Lambda_{L O}$ where $\left|a^{L O}\left(\Lambda_{L O}\right)\right|=1$ given in eq. (3.3) has an important meaning as an upper bound of strong effects.

One way to describe such effects beyond fixed order unitarity violation in a phenomenological fashion is via the use of unitarization methods. One of these prescription specially important is the so-called Inverse Amplitude Method (IAM), which maintains the proper analytical structure of fixed order calculation with the correct branching cuts and without the need of extra parameters. The method is derived from dispersion relation and can be regarded as a resumation of $s$-channel bubble diagrams $[9,10]$. For certain values of the chiral coefficients, the unitarized amplitudes present poles that can be interpreted as dynamically generated resonances. The saturation of unitarity via resonances is indeed the expectation for typical strong dynamics $[11,12]$.

The IAM defines the unitarized amplitudes $a_{I J}^{I A M}(s)=a_{I J}^{(0)}(s) /\left(1-\frac{a_{I J}^{(1)}(s)}{a_{I J}^{(0)}(s)}\right)$ which for low energies restore the chiral amplitudes while fully satisfying the unitarity condition. From the denominator of the IAM amplitudes a mass and a running width can be extracted and related to the Wilson coefficient by $L_{A}\left(M_{A}\right)=-0.0229556+0.181548 / v_{A}^{2}$ with $v_{A} \equiv \frac{M_{A} \sin \theta}{\mathrm{TeV}}$.

We can now compare the amplitudes derived with the IAM with the ones computed from the full $\sigma$ Lagrangian eq. (2.3) and access the possible values of its parameters analyzing the unitarity of the amplitudes.

For large values of $v_{A} \gtrsim 1$ the growing behavior of the LO piece renders difficult for a resonance to unitarize the amplitude. This fact is illustrated in the left hand panel of fig. (1) where we show the $a_{A 0}(s)$ amplitudes for 3 values of $v_{A}=0.5,1,1.5$, using the IAM unitarization model (solid curve), the fixed width $\sigma$ resonance (dashed) or a running width, $\Gamma_{f i x} \rightarrow \frac{\Gamma_{r u n}}{M} s$, (dotted). The value of the coupling is fixed to $g_{\sigma}=0.63\left(g_{\sigma}=\kappa^{\prime} / 2\right)$. It can be seen that values of $M_{\sigma}$ too close or larger than unitarity violation scale, $M_{\sigma} \sim \Lambda_{L O}$, prevent any meaningful use of resonant propagation and a broad continuum appears instead. Moreover, close to the peak the running width approach slightly ameliorates the lineshape description.

Similarly, large couplings can also jeopardize the resonant description and violate unitarity. Extra contributions to the width can dump down and unitarize the amplitude, but nevertheless not helping in the description of the lineshape. In the right hand panel of fig. (1) we show the $a_{A 0}(s)$ amplitudes for 3 values of $g_{\sigma}$ using the IAM unitarization model (cyan), a fixed width (solid) and a running width (dashed). We set $v_{A}=1$.

\section{Experimental signatures at Future Colliders}

We found in previous sections that the dynamically inspired parameters of the effective Lagrangian are $g_{\sigma} \sim 0.63$ and $M_{\sigma} \lesssim 1.2 \mathrm{TeV} / \sin \theta$. 

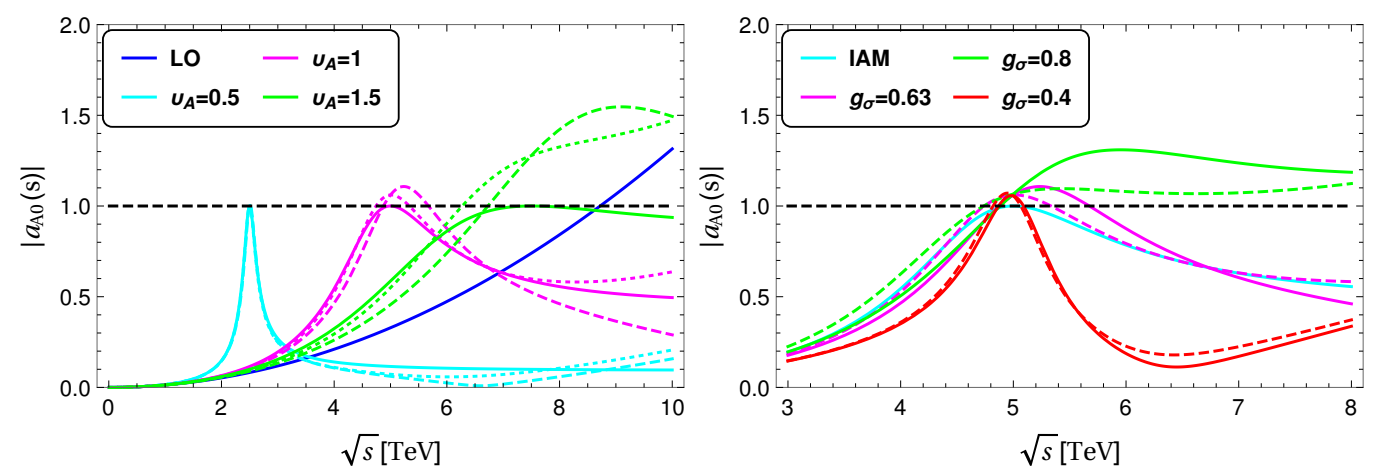

Figure 1: Absolute value of partial wave amplitudes $a_{A 0}^{0}(s)+a_{A 0}^{\sigma}(s)$ for, on the left: $v_{A}=0.5,1,1.5$, using the IAM unitarization model (solid curve), a fixed width $\sigma$ resonance (dashed) and a running width (dotted), and on the right: $g_{\sigma}=0.4,0.63,0.8$, using a fixed width (solid curve) and a running width (dashed) compared to the IAM (cyan).

Due to the intrinsic high compositeness scale of $\mathrm{CH}$ models for $\sin \theta \lesssim 0.2$, these typical strong effects will be observable more likely at a future $100 \mathrm{TeV}$ machine than at the LHC.

In proton-proton collisions, VBS is embedded in more complicated processes where a quark in each proton emits a gauge boson, $V$. These scatter among themselves and produce two $V$ s along with the 2 extra remnant jets in the forward-backward region of the detector. The $V$ s subsequently decay into jets and/or leptons. This process has been studied in the context of CH models [13, 14].

The goal we will pursue here is to assess the possibility to observe the scalar resonance considering only the simplest and cleanest VBS channel where $2 Z$ decay into leptons, $p p \rightarrow j j Z Z \rightarrow$ $j j 4 \ell$. The only relevant backgrounds are SM electroweak $Z Z j j$ and QCD $Z Z+$ jets production. Other VBS channels, $W W, W Z$ and other decay channels will definitely improve the discriminant power here presented [15]. Composite vectors and non-resonant scenarios have been studied in [1].

Events for the process $p p \rightarrow j j Z Z \rightarrow e^{+} e^{-} \mu^{+} \mu^{-} j j$ have been simulated at $\mathrm{LO}$ and showered with the multi-purpose generator SHERPA [16]. Besides the $\mathrm{CH}$ scenario described above, we produced events for the relevant backgrounds: SM EW ZZjj, and the QCD ZZ+jets, merged up to the second jet at LO accuracy. Basic kinematical cuts mimicking detector coverage and typical selection cuts to enhance VBS topology have been implemented. The details of the event production and analysis can be found in Ref [1].

For the statistical assessment we performed a simple counting experiment analysis and computed the probability to exclude the SM assuming one of the $\mathrm{CH}$ scenarios describes Nature. We call this probability $1-\beta$. We assume the probability distributions to follow a Poisson distribution with mean value smeared by a systematic flat error $\varepsilon$ ranging till $40 \%$ to account for scale uncertainty and EW corrections in particular.

In fig. (2) (a) we present the invariant mass of the reconstructed $\mathrm{ZZ}$ system at a $100 \mathrm{TeV}$ collider for the resonant scenarios listed in the legend of the figure. In fig. (2) (b) the corresponding $1-\beta$ are shown. We note a good probability $1-\beta>50 \%$ even for $\sin \theta=0.15$.

At the LHC the situation is more complicated. However, nothing prevents that some dynamical mechanism produces a lighter state. We found nevertheless that even for an optimistic scenario with $g_{\sigma}=0.8, M_{\sigma}=4 \mathrm{TeV}$ and $\sin \theta=0.2$, the effective cross section is only $\sigma=2.9 \times 10^{-4} \mathrm{ab}$. 


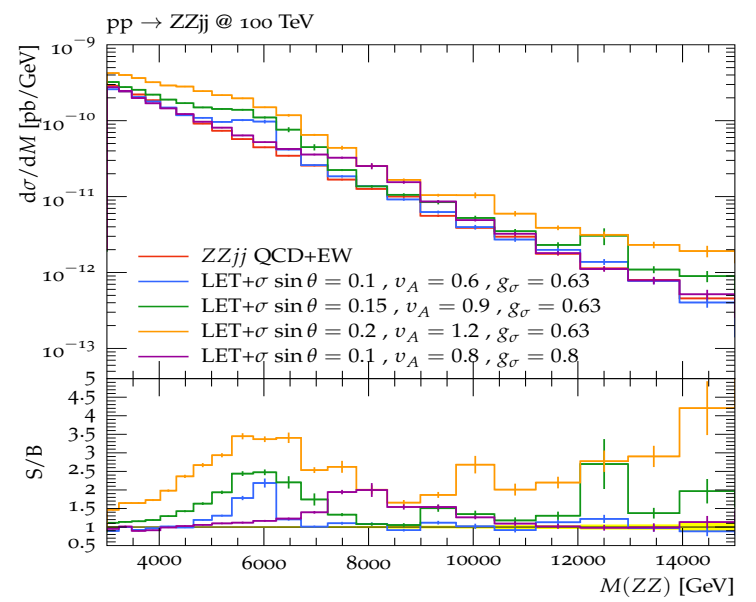

(a)

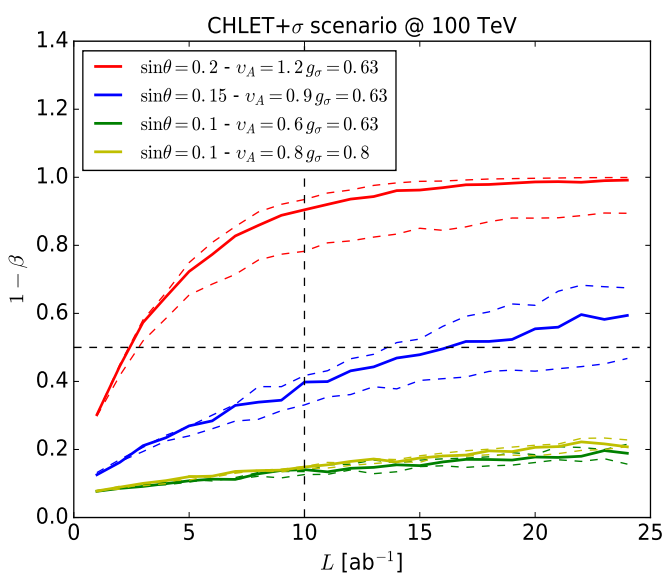

(b)

Figure 2: In (a) the ZZ system reconstructed invariant mass distribution in the $\sigma$ resonant excess scenarios and in (b) the corresponding $1-\beta$.

Including all the other VBS channels is important for this search. Another source of improvement could come from the mixing of $\sigma$ with the Higgs, which at this mass could give some small gluon fusion contribution. Further more detailed study is required.

\section{Conclusions}

In this report we have shown the implications of GBS unitarity in the scalar spectrum of $\mathrm{CH}$ scenarios.

We have especially made definite predictions for the possible range of the mass of an eventual $\sigma$-like composite scalar resonance, which can be described as a Breit-Wigner peak only if $M_{\sigma} \lesssim$ $1.2 \mathrm{TeV} / \sin \theta$. Heavier than that, the LET growing behavior overcomes and dilutes any possible peak, making the strong VBS signal more like a continuum.

Inspired by models of unitarization, which saturate unitarity and provide good description of pion-pion and pion-kaon scattering data, we estimate the parameters of the $\mathrm{CH}$ effective description.

The predictions from the analysis of GBS amplitudes lead to specific signatures at experimental set-ups in colliders. The potential to observe strong VBS in $\mathrm{CH}$ scenario in the simplest channel $p p \rightarrow j j Z Z \rightarrow j j 4 \ell$ has been provided. The results at the $100 \mathrm{TeV}$ collider are promising. The motivated resonant scenario with $\sin \theta=0.15$ and $g_{\sigma}=0.63$ would probably be detected with $L \sim 15 \mathrm{ab}^{-1}$. For lower values of $\sin \theta$ other VBS channels must be considered to enhance the observability potential. At the LHC the situation is more complicated even for very optimistic scenarios, and a more detailed study including other VBS channels and gluon fusion production must be considered. 


\section{References}

[1] D. Buarque Franzosi and P. Ferrarese, Implications of Vector Boson Scattering Unitarity in Composite Higgs Models, 1705.02787.

[2] J. Galloway, J. A. Evans, M. A. Luty, and R. A. Tacchi, Minimal Conformal Technicolor and Precision Electroweak Tests, JHEP 10 (2010) 086, [1001.1361].

[3] G. Cacciapaglia and F. Sannino, Fundamental Composite (Goldstone) Higgs Dynamics, JHEP 04 (2014) 111, [1402.0233].

[4] G. Ferretti, Gauge theories of Partial Compositeness: Scenarios for Run-II of the LHC, 1604.06467.

[5] A. Arbey, G. Cacciapaglia, H. Cai, A. Deandrea, S. Le Corre, and F. Sannino, Fundamental Composite Electroweak Dynamics: Status at the LHC, 1502.04718.

[6] S. R. Coleman, J. Wess, and B. Zumino, Structure of phenomenological Lagrangians. 1., Phys. Rev. 177 (1969) 2239-2247.

[7] C. G. Callan, Jr., S. R. Coleman, J. Wess, and B. Zumino, Structure of phenomenological Lagrangians. 2., Phys. Rev. 177 (1969) 2247-2250.

[8] G. Cacciapaglia, H. Cai, A. Deandrea, T. Flacke, S. J. Lee, and A. Parolini, Composite scalars at the LHC: the Higgs, the Sextet and the Octet, JHEP 11 (2015) 201, [1507. 02283].

[9] A. Dobado and J. R. Pelaez, A Global fit of pi pi and pi K elastic scattering in ChPT with dispersion relations, Phys. Rev. D47 (1993) 4883-4888, [hep-ph/9301276].

[10] R. L. Delgado, A. Dobado, and F. J. Llanes-Estrada, Unitarity, analyticity, dispersion relations, and resonances in strongly interacting $W_{L} W_{L}, Z_{L} Z_{L}$, and hh scattering, Phys. Rev. D91 (2015), no. 7 075017, [1502.04841].

[11] J. Gasser and H. Leutwyler, Chiral Perturbation Theory to One Loop, Annals Phys. 158 (1984) 142.

[12] G. Ecker, J. Gasser, H. Leutwyler, A. Pich, and E. de Rafael, Chiral Lagrangians for Massive Spin 1 Fields, Phys. Lett. B223 (1989) 425-432.

[13] A. Ballestrero, G. Bevilacqua, D. Buarque Franzosi, and E. Maina, How well can the LHC distinguish between the SM light Higgs scenario, a composite Higgs and the Higgsless case using VV scattering channels?, JHEP 11 (2009) 126, [0909.3838].

[14] R. Contino, D. Marzocca, D. Pappadopulo, and R. Rattazzi, On the effect of resonances in composite Higgs phenomenology, JHEP 10 (2011) 081, [1109.1570].

[15] B. Jager, L. Salfelder, M. Worek, and D. Zeppenfeld, Physics Opportunities for Vector-Boson Scattering at a Future 100 TeV Hadron Collider, 1704.04911.

[16] T. Gleisberg, S. Hoeche, F. Krauss, M. Schonherr, S. Schumann, F. Siegert, and J. Winter, Event generation with SHERPA 1.1, JHEP 02 (2009) 007, [0811. 4 622]. 